\title{
An Overview of Information Technology Standardization Activities Related to Additive Manufacturing
}

\author{
Byoung Nam Lee ${ }^{1}$ Eujin $\mathrm{Pei}^{2}$ Jumyung $\mathrm{Um}^{3}$ \\ 1 Electronics and Telecommunications Research Institute, Daejeon, South Korea \\ 2 Institute of Materials and Manufacturing, Brunel University London, London, UK \\ 3 SmartFactorKL, Trippestadter 122, Kaiserslautern, Germany
}

\begin{abstract}
As Additive Manufacturing (AM) technologies and connected machines are ever more widespread across most industries, there is a need for a better understanding about the standardization activities related to Information Technology (IT) within the scope of AM. The purpose of this paper is to provide an overview of existing standardization activities related to IT aspects of AM, including but not limited to data transfer and exchange. The paper covers the IT standardization activities in organizations including ISO TC261, ASTM International, ISO TC 184, ISO/IEC JTC 1, Web3D Consortium, IEC committees, IEEE committees, 3MF Consortium and Khronos who have the most active and ongoing projects. From this study, it can be observed that the manufacturing sector has generated the majority of standardization work, followed by industry R\&D areas and the medical field. This paper also discusses two future IT standards for AM service platforms and for the medical industry.
\end{abstract}

Keywords: Additive Manufacturing, Standardisation, Data Transfer, AMF, STEP-NC

\section{Background}

According to ISO/ASTM 52900:2017, Additive Manufacturing (AM) is a process of joining materials to make parts from 3D model data, usually layer upon layer, as opposed to subtractive manufacturing and formative manufacturing methodologies (ISO/ASTM, 2017). A key advantage is that AM eliminates the need for tooling, such as molds and dies that can make production expensive. AM has the potential to improve supply chain efficiency significantly, reduce time to market, enable mass customization and support environmental sustainability (Ford, 2014). The increased freedom to develop and redesign prototypes and parts without slowing down or adding to production costs also enables a lean and effective product development process. Similarly, the ability of seamless data exchange between AM machines and CAD standard files improves production planning within a digital factory where connected systems can accurately predict the time and material requirements needed to build parts before they are sent to the machine.

The AM process begins from a 3D CAD model or data from a 3D scanner containing geometrical information. Once the design is ready, a user typically saves the design into a geometric data format such as STL (Standard Tessellation Language) file, in which the software transforms the entire surface of the digital model into a mesh of triangles. In the next step, a software known as a slicer, converts the mesh into a 
series of commands to create the model layer by layer. Depending on the AM process, the commands may be used to activate a light source to harden or fuse the material or to control a nozzle to extrude a pre-determined amount of material at a given location. At present, during the AM process, STL is used as a data format that bridges CAD to the AM machine. The usage of STL files, however, results from the historical development of $\mathrm{AM}$ and has been the subject of much debate due to poor geometrical accuracy.

The problems with STL have led to several attempts to change it. For instance, Carleberg (1994) was one of the first to suggest STEP (Standard for the Exchange of Product model data) as a suitable alternative. Many AM controllers use a format called CLI (Common Layer Interface) to represent the section, essentially a polyline boundary and cross-hatched interior, to control the solidification process. In today's industrial context, the machine tool format known as G-code has been used for control. G-code originated in the 1950s for controlling CNC machines and pen plotters and can describe only paths for tool movements and simple machine functions. The latest alternative is ISO 14649 (ISO 14649 - Industrial automation systems and integration - Physical device control - Data model for computerized numerical controllers) which was developed to replace G-codes for control. ISO 14649 is better known as STEP-NC and was created to introduce interoperability into manufacturing enterprises so as to meet the challenge of responding to production on demand (Yusof, 2010). STEP-NC uses the same format for additive and other processes and so allows the same format to be used for post-processing such as removing residual support material and for inspection. Figure 1 shows the example of comprehensive representation of all relevant data of $\mathrm{AM}$ process. In spite of these advantages of using updated standards related Information Technology (IT), primitive standards, such as STL and G-Code, are still utilized in commercial machines.

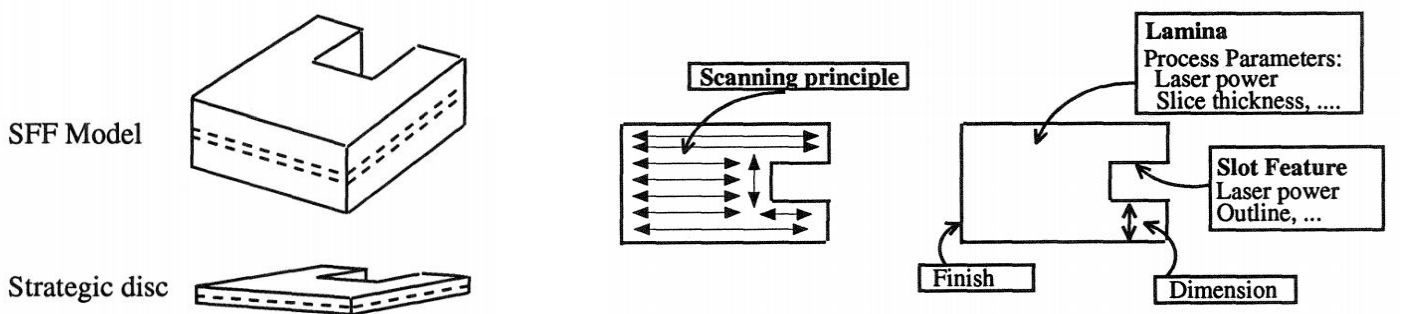

Figure 1. General STEP-based model composed of strategic discs accompanied by process parameter and scanning principles (Carleberg 1994)

Another input data of AM process, in addition to STL, are material, machine configuration, and process parameters. The STL format does not provide sufficient data for tool paths. For example, Selective Laser Melting processes are able to incorporate a wide spectrum of materials. The uniform size of the powder is the one of the critical factors to determine the quality of the parts. The material influences the process parameters and the print resolution and as a result, the melting temperature determines the final result. During the process, the temperature of the bed and the operating environment as well as the laser path, along with the slice profile and tool 
path of the geometric data is defined by the machine operator. The print speed and support structures are also considered as the process parameters. All of these information are fundamental information as part of the AM process. With regards to standardization, vendor specific software still dominate the market and therefore the input parameters are not consistent due to the rapid development of AM technology. The aim of this paper is to provide a current overview of IT standards concerning AM processes.

As AM technologies and connected machines are ever more widespread across most industries, there is a need for a better understanding about the standardization activities related to Information Technology within the scope of AM. Over the last few years, projects focused on the standardization of AM have been carried out mainly by the International Organization for Standardization (ISO) and the American Society for Testing and Materials (ASTM). However, with the proliferation of AM across domains and industry sectors, other standardisation bodies have identified gaps in industry best practices and actions have been carried out by different organizations. For example, in terms of data transfer, Lee, Kaneko and Mekuria (2015) reported that the Moving Picture Experts Group (MPEG) have started standardization activities to realize a common data interface and file format geared towards the specific requirements of $\mathrm{AM}$. They claimed that $3 \mathrm{D}$ representation using voxels with color and material information can be based on point clouds defined by MPEG-4 which also supports compression and transport of 3D assets to reduce the bandwidth and sharing time in a network environment with a MPEG-V engine. Within the scope of manufacturing, other researchers reported the need to improve the final quality of parts made by AM and they proposed a STEP-based numerical control (STEP-NC) process planning approach where the geometric data would also contain information necessary for surface quality accuracy. As more standardization activities and published standards are expected over the next few years, there is a current problem of information becoming fragmented and this paper aims to provide a single point of reference. It is hoped that the paper will enable researchers, industrialists and end-users to gain broader awareness and to utilize this information for future work, as well as to provide a foundation for future useful standards. In the long term, coordinated work with other standards committees and SDOs focused on CAD, IT and $\mathrm{AM}$ technologies will enhance the coherence, capabilities and interoperability of emerging technologies.

\section{Information Technology Standardization Activities}

Standards have to satisfy the needs of the different groups such as industrial, trade, and consumer sectors. As early as the 1990s and 2000s, scholars have examined the need for informational requirements for AM (then known as rapid prototyping and layered manufacturing), proposing a new Application Protocol for the international standard ISO 10303 to specifically handle layered manufacturing information, whereby the official title of the standard is "Automation systems and integration Product data representation and exchange', informally known as "STEP" which 
stands for "Standard for the Exchange of Product model data". ISO 10303 can represent 3D objects in Computer-aided design (CAD) and can be used to exchange data between CAD and computer-aided manufacturing (Pratt et al., 2002). An even earlier paper by Kumar and Dutta (1997) provided an assessment of data formats for layered manufacturing, including .STL, .STH, .CFL and .RPI in which the .RPI format allows more complex representations to be stored with relevant geometrical, topological and process information. The paper proposed STEP and G-WoRP as effective formats that could retain $C A D$ and slicing information for $A M$ and also agreed that industry standards were urgently needed. Since then, most experts agree that the lack of standards is one of the underlying reasons that has prevented the widespread adoption of AM (Monzon et al, 2015). Following an extensive review of existing literature, we compiled data from ISO and IEC Committees, various web resources and SDO documents. In addition, the Technology Trend Report on 3D Printing and Scanning (ISO/IEC JTC 1 N13177) document was used to outline AM standardization activities occurring in the following committees and organizations. This paper will cover the IT standardization activities related to AM within ISO TC261, ASTM International, ISO TC 184, ISO/IEC JTC 1, Web3D Consortium, IEC committees, IEEE committees, 3MF Consortium and Khronos who have the most active and ongoing projects. Table 1 provides a broader cover of AM Standardization Activities related to IT.

Table 1. List of AM Standardization Activities related to IT

\begin{tabular}{|l|l|}
\hline Committee & Committee Name \\
\hline ISO TC 261 & Additive Manufacturing \\
\hline ISO TC 61 & Plastics \\
\hline ISO TC 106 & Dentistry \\
\hline ISO TC 119 & Powder Metallurgy \\
\hline ISO TC 171/SC 2 & Document File Format \\
\hline ISO TC 172/SC 9 & Electro-Optical Systems \\
\hline ISO TC 184/SC 1 & Industrial Cyber and Physical Manufacturing Systems \\
\hline ISO TC 184/SC 4 & Industrial Data \\
\hline IEC TC 62 & Electrical Equipment in Medical Practice \\
\hline IEC TC 76 & Optical Radiation Safety and Laser Equipment \\
\hline IEC TC 108 & $\begin{array}{l}\text { Safety of Electronic Equipment within the Field of } \\
\text { Audio/Video, Information Technology and Communication } \\
\text { Technology }\end{array}$ \\
\hline IEC TC 119 & Printed Electronics \\
\hline IEEE-ISTO & Printer Working Group \\
\hline IEEE C3DP & Consumer 3D Printing Working Group \\
\hline IEEE 3DMA & 3D Based Medical Application Working Group \\
\hline ASTM F42 & ASTM Committee on Additive Manufacturing Technologies \\
\hline ASTM E57 & ASTM Committee E57 on 3D Imaging Systems \\
\hline 3MF & 3MF Consortium \\
\hline DICOM & Digital Imaging and Communications in Medicine \\
\hline
\end{tabular}




\begin{tabular}{|l|l|}
\hline Khronos & Khronos 3D Format Working Group \\
\hline CIE Division 8 & $\begin{array}{l}\text { CIE (International Commission on Illumination) Division 8 } \\
\text { (Image Technology) }\end{array}$ \\
\hline Web3D & Web3D Consortium \\
\hline JTC 1/SC 24 & $\begin{array}{l}\text { Computer Graphics, Image Processing and Environmental } \\
\text { Data Representation }\end{array}$ \\
\hline JTC 1/SC 28 & Office Equipment \\
\hline JTC 1/SC 29/WG 11 & $\begin{array}{l}\text { Coding of Audio, Picture, Multimedia and Hypermedia } \\
\text { Information }\end{array}$ \\
\hline
\end{tabular}

\subsection{ISO TC 261}

ISO TC 261 covers standardization activities in the field of AM concerning processes, terms and definitions, process chains (hardware and software), test procedures, quality parameters, supply agreements and all kind of fundamentals. There are several Working Groups (WG) and Joint Working Groups within the parent Technical Committee (TC) and in particular, ISO TC 261/WG 4 focuses on Data and Design aspects of AM. To date, several standards have been published, of which three documents present information noteworthy in the field of IT, namely ISO 17296-4:2014: Additive manufacturing - General principles - Part 4: Overview of data processing; ISO/ASTM 52915:2016: Specification for additive manufacturing file format (AMF) Version 1.2; and ISO/ASTM DIS 52910 Standard Practice - Guide for Design for Additive Manufacturing. ISO/ASTM 52900:2015: Additive manufacturing General principles - Terminology is a useful source of reference for all terms and definitions associated with AM. For example, the standards document explains the Additive Manufacturing File Format; the extensible markup language; the initial graphics exchange specification (IGES); Product Data Exchange Specification (PDES); standard for the exchange of product model data (STEP) and the STL file formats (ISO/ASTM, 2017).

ISO 17296-4:2014 covers principal considerations which apply to data exchange for AM. It specifies the terms and definitions that enable information to be exchanged, describing geometries or parts such that they can be additively manufactured. The data exchange method outlines the file type, the data enclosed formatting of such data and what this can be used for. The standardization document is aimed at users and producers of AM processes and associated software systems. It applies wherever AM processes are used, and to the following fields in particular: production of additive manufacturing systems and equipment including software; software engineers involved in CAD/CAE systems; reverse-engineering systems developers; and test bodies wishing to compare requested and actual geometries.

ISO/ASTM 52915:2016: Specification for additive manufacturing file format (AMF) Version 1.2 provides the specification for AMF which is an interchange format that was developed to address current and future needs of AM technology. As AM processes have evolved from producing single material, homogeneous objects to 
fabricating parts in full color with functionally graded materials and microstructures, there is a growing need for a standard interchange file format that can support these features. The AMF format is machine independent where by it is able to describe an object in such a general way that any machine can build it to the best of its ability. It is resolution and layer thickness independent and does not contain information specific to any one manufacturing process or technique. It was also designed to be easy to implement and understand where it can be read and debugged in a simple text viewer to encourage comprehension and adoption. The AMF format scales well with an increase in part complexity and is able to retain smooth curved surfaces when there is a requirement for fabrication with very high resolution. The AMF format is backwards compatible and it can be converted to STL for use on legacy systems, although advanced features will be lost. This format maintains the triangle-mesh geometry representation to take advantage of existing optimized slicing algorithms and code infrastructure already in existence. Lastly, to remain useful in a rapidly changing industry, AMF is easily extensible while remaining compatible with earlier versions and technologies. This allows new features to be added as advances in technology warrant, while still working flawlessly for simple homogeneous geometries on the oldest hardware.

ISO/ASTM DIS 52910 Standard Practice - Guide for Design for Additive Manufacturing (previously known as ISO/DIS 20195) includes section 7.10 about File Source - CAD vs. CT in which there are a number of file sources that can be used to generate STL and AMF files such as from scanned data and CAD. It points out that errors can occur due to CT slice scan thickness and resolution, point cloud quality from scanners, and similar resolution limitations from other sources of scanned data, in particular useful for designers, engineers and AM machine operators to understand the quality of files being used to design components intended for AM.

\section{$2.2 \quad$ ASTM Committee F42}

The ASTM Committee F42 on Additive Manufacturing Technologies was developed to promote knowledge, stimulation of research and implementation of technology through the development of standards for AM. It was created in 2009 and ASTM F42 has led a number of $A M$ standardization initiatives, including the specification of the AMF standard. In 2013, ASTM F42 and ISO Additive Manufacturing Committees agreed on combined efforts under a Partner Standards Developing Organization Agreement (PSDO), in the principle of establishing one set of AM standards to be used all over the world and with a common road map and organizational structure for AM standards. As a result, a number of ASTM F42 initiatives are now developed in close collaboration with ISO TC 261, such as ISO/ASTM 52915:2016 Specification for additive manufacturing file format (AMF) Version 1.2 or the ISO/ASTM DIS 52910 Standard Practice - Guide for Design for Additive Manufacturing. Within the organization, F42.04 focuses on Design aspects of AM and covers activities related to data and IT. 


\subsection{ISO TC $184 / S C 1$}

ISO TC 184 covers standardization in the field of automation systems and their integration for design, sourcing, manufacturing, production and delivery, support, maintenance and disposal of products and their associated services, in which standardization areas include information systems, automation and control systems and integration technologies. ISO TC 184/SC 1 deals with Physical device control with the scope "standardization of the control, the interface, the interoperability, the definition and the integration in the area of industrial cyber and physical manufacturing systems covering all manufacturing technologies. This includes but is not limited to symbols, codes, formats, axis and motion nomenclature, command languages and related system aspects, programming methods, simulation, information and data exchange, definition and integration of data and definition of data models for manufacturing systems". There are two WGs, in which WG 7 deals with data modeling for integration of physical devices; and WG 9 deals with interfaces between manufacturing systems. The main involvement with $\mathrm{AM}$ comes through the work of WG 7 on the modern control standard, STEP-NC. SC 1/WG 7 has worked on and produced a number of standards in the area of machine tool control. Machine tool control was first standardized as ISO 6983, which dealt with numerical control in the early days of computer development. Originally developed in the 1960s, various control developers added non-standardized extensions which has led to the current state where part programs became specific for controllers rather than being standard. In view of this, as well as the advances in computing power and general sophistication of machine tools, various interested stakeholders, led by Siemens, developed a new philosophy for machine tool control, called STEP-NC and later standardized as ISO 14649. This new philosophy involves moving CAD data to the machine tool controller and enables a whole new generation of advanced, intelligent control. The standard file format includes information about what is to be made rather than how to make it, as with ISO 6983. STEP-NC is also characterized as a "micro process plan". A draft of the AM part has been developed and is currently being revised for publication. The STEP-NC standards have been used by ISO TC184/SC 4 Digital Manufacturing WG in what is termed as an "Application Reference Model". Although the standard provides many advantages for traditional machining processes such as milling and turning, it offers many more for innovative processes such as EDM (Electrical discharge machining) and AM. The STEP-NC part includes common information, such as the shape, information on materials and detailed control information. It also allows the development of intelligent controllers to allow AM to move into a 'plug-and-play' market for unsophisticated users (Um et al. 2017). Perhaps more importantly, it allows developers of new machines and materials to optimize control for their processes. The work of SC 1 in the development of new control platforms, in WG 7 combined with WG 9, is an important step in the use of AM as part of the general process chain.

\subsection{ISO TC $184 / S^{2} 4$}

ISO/TC 184/SC 4 develops and maintains ISO standards that describe and manage 
industrial data throughout the life of the product. Its work includes modeling of industrial, technical and scientific data to support the exchange, sharing and long term archiving of product data. Among them, ISO 10303 is a standard for the computer-interpretable representation and exchange of product manufacturing information. The title is "Automation systems and integration - Product data representation and exchange", informally known as "STEP" that stands for "Standard for the Exchange of Product model data" and has been in industrial use since 1997. ISO 10303 can represent 3D objects in CAD and related PLM and support information throughout the entire product lifecycle, and is under continuous extension to support growing industry requirements for increased functionality, including systems engineering, simulation and electronics. It is delivered as a series of application protocols, each of which provides a consistent information model to address a particular industrial data requirement. The design information in STEP provides the basic definition for multiple manufacturing processes, including AM. The latest versions of the design application protocols include the option to deliver tessellated geometry as an option for manufacturing, although the direct use of the exact geometry held in STEP along with the associated manufacturing information and tolerances is obviously preferred. Since 2007, STEP Part 238 has provided the capability to drive digital manufacturing systems direct from the design model into the control system of the machine tool. Other STEP documents provide production planning functions. Based on operational experience and innovation, a new edition of AP 238 is being developed to support new classes of manufacturing tools, such as AM and combined additive/subtractive manufacturing devices. ISO TC 184/SC 4 adopted a resolution to establish a new working group for "Digital Manufacturing" (ISO TC 184/SC 4/ WG 15) to ensure a fully integrated approach to digital manufacturing, based on the standardized design model and the experience with AP 238 that would comprise of AM systems. The scope of the new WG is to identify and where necessary develop a coherent set of Industrial Data Standards to maximize the efficiency for the realization of digital products including the areas of digital control, digital planning, digital monitoring, digital simulation, digital validation and digital inspection, in full cooperation with other standards development organizations. This will include interfaces to groups such as ISO TC 29/WG 3 for cutting tools, ISO TC 261 for AM and ISO TC 299 for robotics. ISO TC 184/SC 4 is also responsible for a variety of standardization activities for $3 \mathrm{D}$ visualization of geometry and other product characteristics including COLLADA (PAS 17506) and ISO 14306, based on the JT specification. There are also plans to create a new JWG to manage these standards and the interface from STEP to other 3D formats suitable for consumption by tools for visualization, $A M$ and other functions that do not require a full CAD model or toolset.

\subsection{ISO/IEC JTC 1/SC 24}

ISO/IEC JTC 1 is a joint technical committee of the International Organization for Standardization (ISO) and the International Electrotechnical Commission (IEC). ISO/IEC JTC 1 is the standards development environment for experts to develop worldwide Information and Communication Technology (ICT) standards for business 
and consumer applications, providing standards approval for integrating diverse and complex ICT technologies. ISO/IEC JTC 1/SC 24 covers the standardization of interfaces for information technology based applications related to computer graphics, image processing, environmental data representation, support for the augmented reality continuum (ARC), and interaction with and visual presentation of information. Within SC24, there are 4 Working Groups of interest, comprising WG 6 (Mixed and augmented reality (MAR) presentation and interchange); WG 7 (Image processing and interchange); WG 8 (Environmental data representation); and WG 9 (Mixed and augmented reality (MAR) concepts and reference model). ISO/IEC JTC 1/SC 24/WG 6 initiated the standardization of Extensible $3 D(X 3 D)$ that is related to $A M$ and $3 D$ scanning in collaboration with the Web3D consortium. The ISO/IEC 19775 (Extensible 3D) standard is already used in AM as a 3D file format to support AM services by Shapeways, Thingiverse and browser-based 3D design and online modelers such as TinkerCad. There are converters available from ISO 10303-203 and 10303-214 STEP files to X3D for lightweight visualization and printing.

\section{$2.6 \quad$ ISO/IEC JTC 1/SC 28}

ISO/IEC JTC1/SC28 covers the standardization of basic characteristics, test methods and other related items of products such as 2D printers, AM machines, 3D scanners, copiers, projectors, fax and systems composed of their combinations, excluding such interfaces as user system interfaces, communication interfaces and protocols. The sub-committees include the AG (Advisory Group), WG 1 (New opportunities for office equipment), WG 2 (Consumables), WG 3 (Productivity), WG 4 (Image quality assessment), and WG 5 (Office color). JTC 1/SC 28 is currently investigating consumer/ office areas for AM standardization, but as of now there are no active projects.

\subsection{Web3D Consortium}

Founded in 1997, the Web3D Consortium develops and maintains royalty-free ISO standards for web-based 3D graphics. The Web3D Consortium is an international non-profit SDO supported by a Category A liaison with ISO/IEC JTC 1. The Virtual Reality Modeling Language (VRML97) is the predecessor to X3D and the first ISO/IEC JTC 1 approved standard to be freely published publicly on the Web. Web3D also has formal liaison relationships with the World Wide Web Consortium (W3C), the Open Geospatial Consortium (OGC), Digital Imaging and Communications in Medicine (DICOM) and other groups in order to maximize Web interoperability. When data is presented in an X3D file it can be visualized with X3D players available over all platforms and the geometric data and metadata can be written and read with open, non-proprietary tools. The X3D standard is currently in use in the consumer AM market through online tools and services including the company Shapeways that accept X3D files. In addition to metadata representations and geometric efficiencies, X3D also supports multiple color printing. Online solid modelers allow consumer users to prepare 3D designs using browser-based application, and several of these including TinkerCAD and Clara.io, support exporting a user's design file in X3D format 
for submission to an AM service. Popular archiving services such as Thingiverse and NIH Print Exchange also support the X3D format. X3D includes a document metadata model matching HTML, and also includes a Metadata component which enables embedding of strongly typed metadata anywhere within an X3D scene graph. Current working group efforts are examining addition of a potential Annotation component to facilitate sharable markup and situated display of user metadata annotations. Implementation efforts are especially keen to demonstrate effective integration of ISO metadata libraries suitable for AM, CAD and medical applications. Two distinct types of compression are being established for X3D models. The Shape Resource Container (SRC) work by Fraunhofer IGD provides a variety of geometric compression schemes (polygonization efficiencies, quantization, etc.). Through cooperative work between Web3D Consortium and the Khronos Group, SRC has been fully aligned with the recently released gITF version 2.0. The X3D Working Group is now reviewing additional X3D requirements unmet by gITF version 2.0 to determine further activities. Additionally, cooperative work with the World Wide Web Consortium (W3C) is applying the Efficient XML Interchange (EXI) schema-based compression standard to the X3D XML encoding to support data compaction and decompression performance. Additional cooperative work with $\mathrm{W} 3 \mathrm{C}$ has already applied XML Security capabilities to X3D, including both XML Encryption for privacy and XML Digital Signature for authentication which are expected to support a wide range of use cases for secure AM production of X3D models. Another area of development is that joint work between SC 29 and SC 24 Working Group 9 on the ISO/IEC JTC1 Joint Ad hoc Group (JAhG) Mixed Augmented Reality (MAR) Reference Model, draft ISO/IEC 18039, includes the possible use of AM markers and physical objects within MAR spaces. More importantly, the X3D standard also includes a CAD Geometry Component which supports representation of product assembly structure and face features in X3D scenes. The CAD Interchange Profile defined in the X3D standard also supports distillation of computer-aided design (CAD) data for downstream applications. Multiple conversion routes from STEP (ISO 10303) exchange files to X3D scenes have been identified, including standalone translation software and web based applications. Commercial CAD applications commonly support export to VRML file format which is a subset of X3D and readily converted to X3D files in Classic VRML or XML encoding. There are also SC24 liaison efforts with ISO/TC 184/SC 4 that demonstrate consensus and progress regarding the suitability of creating a combined X3D Profile for CAD, AM and 3D Scanning.

\section{$2.8 \quad$ IEC TC 62}

The International Electrotechnical Commission (IEC) is an international standards organization that prepares and publishes International Standards for all electrical, electronic and related technologies - collectively known as "electrotechnology". IEC TC 62 (Electrical Equipment in Medical Practice) prepares international standards and other publications concerning electrical equipment, electrical systems and software used in healthcare and their effects on patients, operators, other persons and the environment. They cover the use of AM for medical applications such as the 
preparation of anatomical models, customized implants and bio-printing. Within IEC TC 62, SC 62B (Diagnostic Imaging Equipment) and SC 62C (Equipment for Radiotherapy, Nuclear Medicine and Radiation Dosimetry) have a close relationship with DICOM for medical imaging file formats in which the data output may be used for AM to produce medical devices and parts

\section{$2.9 \quad$ IEC TC 76}

IEC TC 76 (Optical Radiation Safety and Laser Equipment) is the leading body on laser standardization, including the high-powered lasers used in industrial and research applications as well as in AM systems. TC 76/JWG 10 is the Joint ISO/IEC Working Group for the safety of lasers and laser equipment in industrial materials processing environments, responsible for maintaining the ISO 11553 series jointly with ISO TC 172/SC 9 - Laser and electro-optical systems.

\section{$2.10 \quad$ IEC TC 108}

IEC TC 108 (Safety of Electronic Equipment within the Field of Audio/Video, Information Technology and Communication Technology) addresses safety aspects of a large range of IT equipment, including AM. Within this Technical Committee, IEC 60950-1 (Safety of IT equipment) and IEC 62368-1 (Safety of audio-visual, information and communication technology equipment) are the main applicable standards series.

\subsection{IEEE-ISTO Printer Working Group (PWG)}

The IEEE Industry Standards and Technology Organization (ISTO) was established in January 1999 as a federation of member alliance programs with the aim of supporting accelerated technology standards development and market adoption for industry. The Institute of Electrical and Electronics Engineers (IEEE) focuses on aspects of electrical and electronic engineering, telecommunications, computer engineering and other allied disciplines. The IEEE is the founding member of ISTO which established the Printer Working Group (PWG) in September 1999 with members including printer and multi-function device manufacturers, print server developers, operating system providers, print management application developers, and industry experts. The IEEE-ISTO PWG currently has two active WGs. The Internet Printing Protocol (IPP) WG is focusing on harmonized $2 \mathrm{D}$ and $3 \mathrm{D}$ Printing (AM); and the Imaging Device Security (IDS) WG is focusing on collaboration with the international Common Criteria community and other external standards bodies. The PWG Standard 5100.12: IPP 2.0, 2.1, and 2.2 was published in October 2015. Notably, the PWG Candidate Standard 5100.21: IPP 3D Printing Extensions v1.0, was published in February 2017 and provides a secure and widely implemented network printing protocol and requires support for files in the 3MF format and recommends support for PDF files containing 3D content encoded in U3D or PRC objects. Taking a step further, the Printer Working Group has developed an extension to the Internet Printing Protocol for AM use. The extension leverages their existing network protocol and Job Ticket formats and existing high-level 3D file formats to describe the objects for production. The IPP 
workgroup has also developed a version of the PWG Semantic Model Print Job Ticket schema for AM to support data exchange and embedding this within common 3D file formats such as 3MF and 3D PDF documents so that the user intent is preserved regardless of the system of transport or workflow.

\subsection{MF Consortium}

Launched in 2015, the 3MF Consortium is an industry group working to define an AM format that will allow design applications to send full-fidelity 3D models to a mix of other applications, platforms, services and printers. The consortium was founded by Microsoft and includes industrial members including HP, Siemens, Dassault Systèmes and Autodesk. The goal is to develop a format that is rich enough to fully describe a model, retain internal information, color, and other characteristics. It aims to be extensible so that it supports new innovations in 3D printing as well as interoperability. The 3D Manufacturing File Format (3MF) aims to offer a compact $\mathrm{XML}$ format with physical dimensions, named materials, and shared vertices.

\subsection{Khronos Group}

COLLADA (COLLAborative Design Activity) is an interchange file format for interactive 3D applications. It is being managed by a non-profit technology consortium, the Khronos Group, and has been adopted by ISO as a publicly available specification known as ISO/PAS 17506:2012 Industrial automation systems and integration COLLADA digital asset schema specification for 3D visualization of industrial data that was published in July 2012. COLLADA defines an XML Namespace and database schema to make it easy to transport 3D assets between applications without loss of information, enabling diverse 3D authoring and processing tools to be combined into a content production pipeline. More importantly, COLLADA is an open standard XML schema that can exchange digital assets among various graphics software applications for portability. COLLADA documents are usually identified with a dae (digital asset exchange) filename extension.

\section{Discussion}

A review of existing IT standardization activities within the scope of AM has shown that generally most activities are focused on the industrial market as well as material and industrial processes. Table 2 provides an outline of ongoing and future activities of IT standards in terms of different application domains. Although there are overlapping areas, it can be observed that the manufacturing sector has generated the majority of standardization work, followed by industry R\&D areas and the medical field. There are opportunities for SDOs to work on future standards to enable greater support for consumer AM services. For example, the development of standards for AM service platforms can help smaller companies and consumers to specify and procure customized AM objects and parts without incurring the cost of investing in expert AM designers, or purchasing and maintaining expensive AM printers.

Table 2. Ongoing and future activities of IT standards related to AM 


\begin{tabular}{|c|c|c|}
\hline \multirow{2}{*}{$\begin{array}{l}\text { Application } \\
\text { Domains }\end{array}$} & \multicolumn{2}{|c|}{ Maturity Level } \\
\hline & Ongoing Activities & Future Activities \\
\hline $\begin{array}{l}\text { Medical } \\
\text { Industry }\end{array}$ & $\begin{array}{l}\text { ISO TC 261/WG 4: NWIP (Medical } \\
\text { Data for Additive Manufacturing) } \\
\text { ISO/IEC JTC 1/SC 24: ISO/IEC } \\
\text { 19775 - X3D Medical Interchange } \\
\text { Profile }\end{array}$ & $\begin{array}{l}\text { IEC TC } 62 \\
\text { ISO/IEC JTC } 1 \\
\text { ISO/IEC JTC 1/SC } 24\end{array}$ \\
\hline $\begin{array}{l}\text { Health \& } \\
\text { Wellness }\end{array}$ & - & $\begin{array}{l}\text { ISO TC } 261 / \text { WG } 4 \\
\text { IEC TC } 124\end{array}$ \\
\hline $\begin{array}{l}\text { Gaming \& } \\
\text { Animation }\end{array}$ & $\begin{array}{l}\text { ISO/IEC JTC 1/SC 24: ISO/IEC } \\
14772 \text { - VRML, ISO/IEC 19774 } \\
\text { H-Anim, ISO/IEC 19775 - X3D }\end{array}$ & $\begin{array}{l}\text { ISO TC 261/WG } 4 \\
\text { ISO/IEC JTC 1/SC } 24\end{array}$ \\
\hline $\begin{array}{l}\text { AR \& VR } \\
\text { Activities }\end{array}$ & $\begin{array}{l}\text { ISO/IEC JTC 1/SC 24: ISO/IEC } \\
\text { 14772 - VRML, ISO/IEC 19775 - } \\
\text { X3D Immersive Profile }\end{array}$ & $\begin{array}{l}\text { ISO TC 261/WG } 4 \\
\text { ISO/IEC JTC 1/SC } 24\end{array}$ \\
\hline Manufacturing & $\begin{array}{l}\text { ISO TC 184/SC 4: draft } \\
\text { 10303-242ed 2, 10303-238, ISO } \\
14306 \\
\text { ISO TC 261/WG 4: ISO } \\
\text { 17296-4:2014, ISO/ASTM } \\
\text { 52915:2016 } \\
\text { ISO TC 184/SC 1: draft ISO/AWI } \\
\text { 14649-17 (20.00) } \\
\text { ISO TC 171/SC } 2 \text { : PDF/E } \\
\text { ISO/IEC JTC 1/SC 24: ISO/IEC } \\
\text { 14772 - VRML, ISO/IEC 19775 - } \\
\text { X3D CAD }\end{array}$ & $\begin{array}{l}\text { ISO TC } 261 / \text { WG } 4 \\
\text { ISO TC } 184 / \text { SC } 1 \\
\text { ISO TC } 184 / \text { SC } 1 / \text { WG } 7 \\
\text { ISO TC } 184 / \text { SC } 4 \\
\text { ISO TC } 184 / \text { SC } 4 / \text { WG } 15 \\
\text { ISO TC } 184 / \text { SC } 4 \text { WG } 16 \\
\text { ISO SMCC } \\
\text { IEC TC } 65 \\
\text { IEC SEG } 7 \\
\text { ISO/IEC JTC 1/SC } 24\end{array}$ \\
\hline $\begin{array}{l}\text { Apparel \& } \\
\text { Fashion }\end{array}$ & - & ISO TC 261/WG 4 \\
\hline Urbanization & $\begin{array}{l}\text { ISO/IEC JTC 1/SC } 24 \text { : ISO/IEC } \\
14772 \text { GeoVRML, ISO/IEC } 19775 \text { - } \\
\text { X3D Geospatial }\end{array}$ & $\begin{array}{l}\text { ISO TC 261/WG } 4 \\
\text { ISO/IEC JTC 1/SC } 24\end{array}$ \\
\hline Transportation & - & ISO TC 261/WG 4 \\
\hline
\end{tabular}




\begin{tabular}{|l|l|l|}
\hline & ISO TC 261/AHG 3 & \\
& ISO TC 261/JG 64 & \\
& ISO TC 261: draft ISO/NP TR & \\
& 52912 (10.99) & \\
Industry R\&D & ISO TC 184/SC 1 & IEC TC 119 \\
& ISO TC 184/SC 4: draft & \\
& ISO/IEC JTC 1/SC 24 \\
& ISO/IEC JTC 1/SC 24 : ISO/IEC & \\
& 14772 - VRML, ISO/IEC 19774 & \\
& H-Anim, ISO/IEC 19775 - X3D & \\
\hline
\end{tabular}

The medical industry is another area that could benefit from IT standards for AM. There are very few existing standard best practices for creation and validation procedures to ensure that medical imaging data can be accurately produced through AM methods. Techniques for medical image segmentation need to be properly optimized and combined according to the image modalities and body parts. A review of existing file formats show that they are not suited for medical-based AM and current references to standardization activities for medical-based AM is largely fragmented. For example, in the digital chain, medical-based AM first involves the optimization of segmented body parts to minimize error which refers to work undertaken by IEC TC 62 and DICOM WG 17. Next, the conversion of 2D to 3D data and reduction of stair stepping relies on standardization work described by ISO TC 261/WG 4 and JTC 1/SC 24. The calibration and validation of the converted $2 \mathrm{D}$ to $3 \mathrm{D}$ data, setting the units and scales, and development of the digital twin refers to the work of ISO TC 261/WG 4, JTC 1/SC 24, JTC 1/SC 25, JTC 1/SC 28/WG 4, JTC 1/SC 38 and IEC TC 62. Finally, the File format creation for the AM process refers to work undertaken by ISO TC 261/WG 4 and the 3MF Consortium. The entire process of a cranioplasty prosthesis medical-based AM method is illustrated in Figure 2, showing the processes involved and the associated standards organizations. It provides evidence of fragmented knowledge and it is timely for coordinated work among standards committees. 


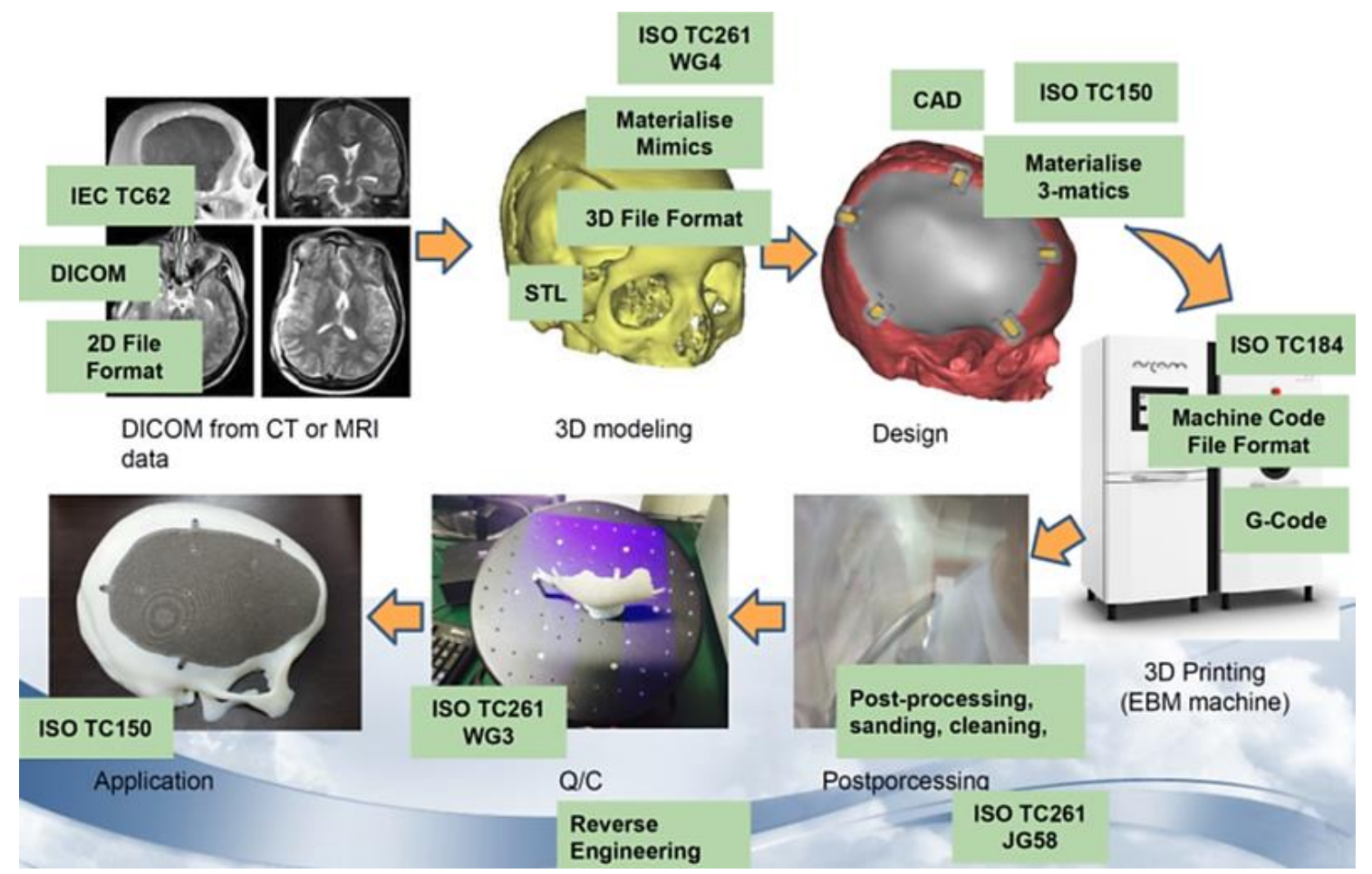

Figure 2. Overview for cranioplasty prosthesis manufactured through $A M$ and the associated standards organizations

\section{Conclusion}

This paper has provided an overview about Information Technology standardization activities related to Additive Manufacturing. It describes the AM process and the data requirements for production. It describes the current state-of-art IT standardization activities related AM occurring within committees and organizations at an international level. The review shows that generally most activities are focused on the industrial market as well as material and industrial processes; and also discusses the two future IT standards for AM that could provide the benefit to consumer and medical industry. It is hoped that this paper will enable readers to gain an awareness of current Information Technology standardization activities related to Additive Manufacturing and to utilize this information for future work.

\section{Acknowledgements}

This paper is a condensed summary from a report presented at ISO/IEC JTC 1 Plenary (October 2017, Vladivostok, Russia) and prepared by the ISO/IEC JTC 1 Study Group under the direction of Resolution 2 (November 2016 JTC 1 Plenary Lillehammer, Norway). The representatives who participated in the work of the ISO/IEC JTC 1 Study Group on 3D Printing and Scanning are listed in Appendix 1.

\section{References}

1. ISO/ASTM (2017) ISO/ASTM 52900:2015 Additive manufacturing - General principles - Terminology. Accessed 30 July 2018 from <<https://www.iso.org/ standard/69669.html>> 
2. Ford, S. (2014) Additive Manufacturing Technology: Potential Implications for U.S. Manufacturing Competitiveness. Journal of International Commerce and Economics. Accessed 14 July 2018 from <<https://ssrn.com/abstract=2501065 >

3. Carleberg, P. (1994) Product model driven direct manufacturing, The SFF Symposium, University of Texas, 270-276

4. Yusof, Y. (2010) ISO 14649 (STEP-NC): New Standards for CNC Machining. International Journal of Integrated Engineering (Issue on Mechanical, Materials and Manufacturing Engineering) Accessed 17 July 2018 from <<http://penerbit.uthm.edu.my/ojs/index.php/ijie/article/view/154>>

5. Lee, S.W., Kaneko, I. and Mekuria, R. (2015) Investigation on 3D Printing. A Report from ISO/IEC JTC1/SC29/WG11 N15303. Accessed on 31 July 2018 from <<https://mpeg.chiariglione.org/sites/default/files/files/standards/docs/ w15303.zip >>

6. Pratt M.J., Bhatt A.D., Dutta D., Lyons K.W., Patil L. and Siriam R.D. (2002) Progress towards an international standard for data transfer in rapid prototyping and layered manufacturing. Computer Aided Design 34: 1111-1121

7. Kumar, V., Dutta, D. (1997) An assessment of data formats for layered manufacturing. Advanced Engineering Software 28(3): 151-164

8. Monzón, M.D., Ortega, Z., Martínez, A. et al. (2015) Standardization in additive manufacturing: activities carried out by international organizations and projects. International Journal of Advanced Manufacturing Technology 76: 1111-1121.

9. Um, J. Y., Rauch, M., Hascoët, J-Y. and Stroud, I. (2017) STEP-NC compliant process planning of additive manufacturing: remanufacturing. The International Journal of Advanced Manufacturing Technology 88. 5-8

\section{Appendix 1 - List of Contributors}

\begin{tabular}{|l|l|}
\hline Name & Affiliation / Organisation \\
\hline Byoung Nam Lee & Convenor JTC1/SG3 \\
\hline Yaeseul Park & Secretary JTC1/SG3 \\
\hline Gilles Thonet & IEC CO \\
\hline Kei Yamashita & JTC 1/SC 23 \\
\hline $\begin{array}{l}\text { Cong Wang, Farid Mamaghani, Don Brutzman, } \\
\text { Roy Walmsley, Ha Jine Kimn, Hwanyong Lee, } \\
\text { Kwan-Hee Yoo, Hyokwang Lee, Myeong Won Lee }\end{array}$ & JTC 1/SC 24 \\
\hline lan Stroud, Jumyung Um & ISO TC 184/SC 1/WG 7 \\
\hline Howard Mason, Soonhung Han & ISO TC 184/SC 4 \\
\hline Eujin Pei, Klas Boivie, Jörg Lenz & ISO TC 261 \\
\hline François Coallier & Canada (SCC) \\
\hline Haibin Li & China (SAC) \\
\hline Johan Wirta & Finland (SFS) \\
\hline Patrick Marchand, Christophe Mouton & France (AFNOR) \\
\hline Brian McAuliffe & Ireland (NSAI) \\
\hline
\end{tabular}




\begin{tabular}{|l|l|}
\hline Satoshi Itoh & Japan (JISC) \\
\hline $\begin{array}{l}\text { Seung Wook Lee, Dongyub Lee, Hongki Cha, } \\
\text { Hyun Jeong Kim, Choon-Woo Kim, Kyu-Won } \\
\text { Shim, Kuyeong Oh, Ji-Man Park }\end{array}$ & Korea(Rep. of) (KATS) \\
\hline Alojz Hudobivn & Slovenia (SIST) \\
\hline Jonathan Porter, Aydin Nassehi & UK (BSI) \\
\hline $\begin{array}{l}\text { Paul Jeran, Christian Page, Michelle Pangborn, } \\
\text { Diane Stephens, Paul Tykodi, Jennifer Garner }\end{array}$ & US (ANSI) \\
\hline Young Lae Moon & $\begin{array}{l}\text { 3D Medical Application Work } \\
\text { Group, IEEE-SA }\end{array}$ \\
\hline Adrian Lannin & 3MF Consortium \\
\hline Lauralyn McDaniel & America Makes \& ANSI AMSC \\
\hline Jérémie Farret & ASTM F42 \\
\hline Pat A. Picariello & ASTM F57 \\
\hline Allan Noordvyk, Justin Ryan & DICOM \\
\hline Vincent Marchetti & Web3D Consortium \\
\hline
\end{tabular}

Appendix 2 - list of acronyms.

\begin{tabular}{|l|l|}
\hline Acronyms & Full name \\
\hline AM & Additive Manufacturing \\
\hline STL & Standard Tessellation Language \\
\hline STEP & Standard for the Exchange of Product Data \\
\hline CLI & Common Layer Interface \\
\hline MPEG & Moving Picture Experts Group \\
\hline STEP-NC & STEP-based Numerical Control \\
\hline SDO & Standards Developing Oganization \\
\hline STH & Surface Triangles Hinted \\
\hline CFL & Cubital Facet List \\
\hline RPI & Rensselaer Polytechnic Institute \\
\hline G-WoRP & Geometric Workbench for Rapid Prototyping \\
\hline 3MF & 3D Manufacturing File Format \\
\hline COLLADA & COLLAborative Design Activity \\
\hline AMF & Additive Manufacturing Format \\
\hline CT & Computer Tomography \\
\hline EDM & Electrical discharge machining \\
\hline X3D & Extensible 3D \\
\hline gITF & GL Transmission Format \\
\hline DICOM & Digital Imaging and Communications in Medicine \\
\hline
\end{tabular}

